\title{
Synthesis and Characterization of Bromoclenbuterol
}

\author{
Ravi Kumar Kannasani*, Srinivasa Reddy Battula, Suresh Babu Sannithi, Sreenu Mula and Venkata Babu VV \\ R\&D Division, RA Chem Pharma Limited, API, Hyderabad, Telangana, India
}

\begin{abstract}
Clenbuterol is a sympathomimetic amine used for breathing disorders as a decongestant and bronchodilator. During the process development of clenbuterol, the process related impurity bromoclenbuterol was identified as a critical impurity along with the final API. The present work describes the synthesis and characterization of this bromoclenbuterol.
\end{abstract}

Keywords: B ICH; Breathing disorders; Impurity; Bromoclenbuterol

\section{Introduction}

Clenbuterol, it is most commonly available as the hydrochloride salt, clenbuterol hydrochloride. Clenbuterol, marketed as Dilaterol, Spiropent, Ventipulmin, and also generically as clenbuterol, is a sympathomimetic amine used for breathing disorders as a decongestant and bronchodilator. People with chronic breathing disorders such as asthma use this as a bronchodilator to make breathing easier. Clenbuterol is a $\beta 2$ agonist with some structural and pharmacological similarities to epinephrine and salbutamol, but its effects are more potent and longerlasting as a stimulant and thermogenic drug. It causes an increase in aerobic capacity, central nervous system stimulation, blood pressure, and oxygen transportation. It increases the rate at which body fat is metabolized while increasing the body's BMR. It is commonly used for smooth muscle-relaxant properties as a bronchodilator and tocolytic. Clenbuterol is also prescribed for treatment of horses, but equine use is usually the liquid form (Figure 1).

Clenbuterol Hydrochloride was first synthesized at Thomae; a Boehringer Ingelheim research facility in Biberach, Germany, in 1967. The synthesis of Clenbuterol Hydrochloride was patented in the United States in 1970. After comprehensive clinical trials, Clenbuterol Hydrochloride was approved for the treatment of reversible airway obstruction in Germany in 1976 and later as a veterinary pharmaceutical for the treatment of bronchiolytic disorders in Germany in 1980. Boehringer Ingelheim markets Clenbuterol Hydrochloride as Spirospent for Human Pharmaceuticals and as Ventipulmin for Veterinary Pharmaceuticals. Clenbuterol Hydrochloride is not approved by the Federal Drug Administration for human use in the United States.

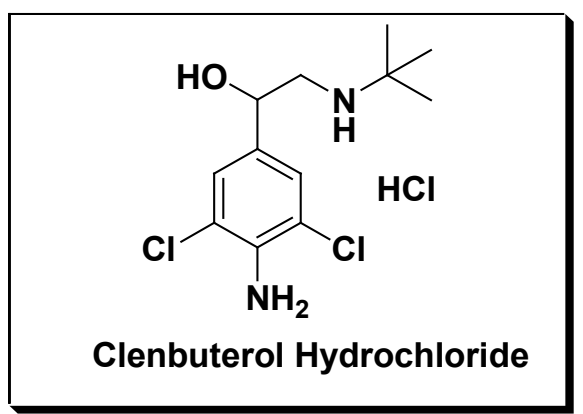

Impurity is defined as any substance coexisting with the original drug, such as starting material or intermediates or those formed, due to any side reactions. The presence of these unwanted chemicals, even in small amounts, may influence the efficacy and safety of the pharmaceutical products. A general scheme is set for the estimation of the impurities in bulk drug substances by the rational use of chromatographic, spectroscopic and analytical techniques. The process-related impurities in an active pharmaceutical ingredient (API) can have a significant impact on the quality and safety of the drug products. It is quite important for "regulatory" aspect of drug approval to provide limitation of "related impurities." Therefore, it is necessary to study the impurity profile of any API and control it during the manufacturing of a drug product. As per the ICH guidelines, impurities which are forming at a level of $\geq 0.10 \%$ with respect to the API should be identified, synthesized, and characterized thoroughly $[1,2]$.

During the process development of clenbuterol hydrochloride in RA Chem Pharma Ltd [3], the process related impurities as per pharmacopoeia (British pharmacopoeia and European pharmacopoeia) are identified and they are listed as 4-amino-3,5-dichloro benzaldehyde (Impurity-A), 1-(4-amino-3,5-dichlorophenyl)-2[(1,1-dimethylethyl)amino]ethanone (Impurity-B), 1-(4-amino3,5-dichloro phenyl) ethanone (Impurity-C), 1-(4-amino phenyl) ethanone (Impurity-D), 1-(4-amino -3,5-dichloro phenyl) -2-bromo ethanone (Impurity-E) and (1RS)-1-(4-amino -3-bromo-5-chloro phenyl) -2-[(1,1-dimethyl ethyl)amino ethanol or bromoclenbuterol (Impurity-F).

The purpose of this work is for determining the impurities of clenbuterol to ensure the quality, efficacy and safety of the active ingredient in final pharmaceutical formulation. Among the six process related impurities, the bromoclenbuterol impurity was identified as a critical impurity and it was formed in the synthesis of clenbuterol hydrochloride API at the time of chlorination of 4-amino acetophenone (Table 1).

The present article describes a simple and facile synthesis for bromoclenbuterol impurity. This may serve as a standard for impurity profiling in drug development and analytical method validations.

\section{Experimental}

The chemicals and solvents were purchased from commercial suppliers and they were used without purification prior to use. ${ }^{1} \mathrm{H}$ NMR spectra were recorded on $400 \mathrm{MHz}$ and ${ }^{13} \mathrm{C}$ NMR spectra were recorded on $100 \mathrm{MHz}$ FT-NMR spectrometers. All chemical shifts

*Corresponding author: Ravi Kumar Kannasani, R\&D Division, RA Chem Pharma Limited, API, Prasanth Nagar, Hyderabad, Telangana, India, Tel: +919000443184 E-mail: kannasani.ravi@rachempharma.com

Received August 25, 2016; Accepted August 29, 2016; Published August 31, 2016

Citation: Kannasani RK, Battula SR, Sannithi SB, Mula S, Babu VVV (2016) Synthesis and Characterization of Bromoclenbuterol. Med Chem (Los Angeles) 6 : 546-549. doi:10.4172/2161-0444.1000397

Copyright: (c) 2016 Kannasani RK, et al. This is an open-access article distributed under the terms of the Creative Commons Attribution License, which permits unrestricted use, distribution, and reproduction in any medium, provided the original author and source are credited. 
are given as $\delta$ value with reference to Tetra methyl silane (TMS) as an internal standard.

\section{1-(4-Amino-3-chlorophenyl)ethanone (7)}

To a stirred solution of $1 \mathrm{~N} \mathrm{HCl}(1500 \mathrm{ml})$ was added 4 -amino acetophenone (1) (200 gm, 1.48 mole) and N-Chloro succinimide (50 gm, 0.37 mole) at room temperature, and stirring continued for $3 \mathrm{hrs}$ at $25-30^{\circ} \mathrm{C}$. After maintenance undissolved material was filtered from the reaction mixture, total filtrate was taken and extracted with ethyl acetate, dried over sodium sulfate and evaporated under vacuum to get crude. Crude material was dissolve in ethyl acetate, titrated with $\mathrm{EA}-\mathrm{HCl}$ and stirred for 15-30 min to get precipitation. The obtained precipitate was filtered and washed with ethyl acetate, and this acidic titration operation was repeated 2 times to get mono chloro compound as solid material, this solid material was neutralized with sodium carbonate solution in aqueous condition and further purified by using recrystlliaztion technique in ethyl acetate to get 68 gm (yield-27\%) 3-chloro-4-amino acetophenone (7) (mono chloro compound), as light brown colored solid with $98.66 \%$ HPLC purity (124 gm of unreacted 4 -amino acetophenone obtained from aqueous layer).

\section{1-(4-Amino-3-bromo-5-chlorophenyl)-2-bromoethanone (8)}

To a stirred solution of 3-chloro-4-amino acetophenone (7) (14 $\mathrm{gm}, 0.082 \mathrm{~mole})$ in chloroform $(140 \mathrm{ml})$ was added bromine $(26.24 \mathrm{gm}$,<smiles>Nc1c(Cl)cc(C=O)cc1Cl</smiles>

Impurity-A<smiles>CC(=O)c1ccc(N)cc1</smiles>

Impurity-D<smiles>CC(C)(C)NCC(=O)c1cc(Cl)c(N)c(Cl)c1</smiles>

Impurity-B<smiles>Nc1c(Cl)cc(C(=O)CBr)cc1Cl</smiles>

Impurity-E<smiles>CC(=O)c1cc(Cl)c(N)c(Cl)c1</smiles>

Impurity-C
Figure 1: Process related impurities of clenbuterol as per pharmacopoeia.
0.164 mole) solution slowly at $25-30^{\circ} \mathrm{C}$ and stirring continued for 6 hrs at same temperature. After completion of the reaction, methanol was added to the reaction mixture and continued the stirring for 30 min at RT. Undissolved material was filtered, the filtrate was distilled up to $50 \%$, remaining mass was cooled to $0-5^{\circ} \mathrm{C}$ and filtered to give 15 gm (yield-55\%) of 1-(4-amino -3-chloro-5-bromo - phenyl) -2-bromo ethanone (8) as light brown color solid with $95.15 \%$ HPLC purity.

\section{2-(Tert-butylamino)-1-(4-amino-3-bromo-5-chlorophenyl) ethanone (9)}

To a stirred solution of 1-(4-amino -3-chloro-5-bromo - phenyl) -2-bromo ethanone (8) (8 gm, 0.024 mole) in chloroform $(50 \mathrm{ml})$ was added catalytic amount of potassium iodide $(0.1 \mathrm{gm}, 0.0006$ mole) and tertiary butyl amine $(5.2 \mathrm{gm}, 0.072 \mathrm{~mole})$ at $0-5^{\circ} \mathrm{C}$ and stirring was continued for $24 \mathrm{hrs}$ at $0-5^{\circ} \mathrm{C}$. After completion of the reaction, undissolved salts were filtered, the filtrate was distilled under vacuum to get crude solid material, which was triturated with hexane to give $6 \mathrm{gm}$ (yield-76\%) of 1-(4-amino-3-chloro-5-bromo phenyl)-2-[(1,1dimethylethyl)amino]ethanone (9) as light pale yellow color solid.

\section{(S) - 2 - ( Tert - butylamino ) - 1 - (4 - a mino-3 - bromo- 5 -} chlorophenyl)ethanol (10)

To a stirred solution of 1-(4-Amino-3-chloro-5-bromo phenyl)2-[(1,1-dimethylethyl)amino]ethanone (9) (6 gm, 0.018 mole) in methanol $(25 \mathrm{ml})$ was added sodium borohydride $(0.7 \mathrm{gm}, 0.018$ mole) at $0-5^{\circ} \mathrm{C}$. After addition, reaction mixture was slowly allowed to come to room temperature and stirred for $10 \mathrm{hrs}$ at $25-30^{\circ} \mathrm{C}$. On completion, reaction mixture was poured in to chilled water, obtained precipitate was filtered, dried and recrystallized in methanol to give $5 \mathrm{gm}$ (yield-82\%) of 1RS-1-(4-amino -3-bromo-5-chloro phenyl) -2-[(1,1-dimethyl ethyl)amino ethanol (or) Bromo clenbuterol (10) as off-white solid. HPLC purity-98.80\%, ${ }^{1} \mathrm{H}$ NMR $\left(\mathrm{CDCl}_{3}\right): \delta 7.35(\mathrm{~d}, 1 \mathrm{H}$, $J=1.2 \mathrm{~Hz}$ ), 7.23 (d, $1 \mathrm{H}, J=1.6 \mathrm{~Hz}), 4.45$ (br s, $2 \mathrm{H}), 4.42$ (dd, $1 \mathrm{H}, J=9.2$, $3.6 \mathrm{~Hz}), 2.84(\mathrm{dd}, 1 \mathrm{H}, J=11.6,3.6 \mathrm{~Hz}), 2.50(\mathrm{dd}, 1 \mathrm{H}, J=12.0,9.2 \mathrm{~Hz})$, $1.10(\mathrm{~s}, 9 \mathrm{H}) .{ }^{13} \mathrm{C} \mathrm{NMR}\left(\mathrm{CDCl}_{3}\right): 140.12,133.93,128.46,126.05,119.16$, $109.08,70.94,50.33,50.05,29.15$. IR $\left(\mathrm{KBr}, \mathrm{Cm}^{-1}\right): 3465.99,3320.19$, 2965.04, 1623.40, 1483.88, 1219.17, 758.77, 630.41. Mass: (m/z)-323.01 $\left(\mathrm{M}^{+2}\right.$ peak).

\section{Results and Discussion}

As per the available literature [4-7], clenbuterol hydrochloride was synthesized from 4-amino acetophenone (Scheme 1). Initially 4-amino acetophenone (1) was reacted with chlorine to afford 4-amino-3,5dichloro acetopheneone (2) which was further reacted bromine to give
Impurity 3,5-dichloro acetophenone by over chlorination reaction (haloform reaction).

\section{Control of impurity}

This impurity can be controlled by using crystallization technique.

Impurity-B It is one of the intermediates in the manufacturing process of clenbuterol. It may This impurity has soluble nature in organic solvents and hence it can be present in the final product if any un reacted material present in the process. be eliminated from the process during crystallization process.

Impurity-C It is one of the intermediates in the manufacturing process of clenbuterol. It may This impurity contains soluble nature in organic solvents and hence be present in the final product if any un reacted material is present in the process. it can be eliminated from the process during crystallization process.

Impurity-D It is one of the key starting materials in the manufacturing process of clenbuterol. This impurity contains soluble nature in acidic water and hence it can It may be present in the final product if any un reacted material is present in the be eliminated from the process during reaction work-up. process.

Impurity-E It is one of the intermediates in the manufacturing process of clenbuterol. It may This impurity contains soluble nature in alcohol and hence it can be be present in the final product if any un reacted material is present in the process. eliminated from the process during reaction work-up.

Impurity-F Mainly this impurity will form from 4-Amino -3-chloro acetophenone (mono chloro Mono chloro impurity contains soluble nature in organic solvent impurity). It is one of the known intermediate in chlorination reaction. In further and is brought under control or eliminated during the crystallization stage it will react with bromine and form 1-(4-amino-3-bromo-5-chlorophenyl)-2- process. bromoethanone. It will cause formation of bromoclenbuterol. 
1-(4-amino-3,5-dichlorophenyl)-2-bromoethanone (3). The obtained bromo compound was reacted tertiary butyl amine to afford 2-(tertbutylamino)-1-(4-amino-3,5-dichlorophenyl)ethanone (4), which was further reduced with sodium borohydride to give clenbuterol base (5) and converted in to hydrochloride salt by using alcoholic $\mathrm{HCl}$ to get clenbuterol hydrochloride (6).

In order to find the control and prevention of process related impurities of clenbuterol, the preliminary efforts were mainly focused on the manufacturing process. Among the six process related impurities, the bromoclenbuterol impurity was identified as a critical impurity because it cannot be eliminated from the API molecule, after formation in the reaction. Bromoclenbuterol and clenbuterol possessed same properties like solubility and crystallization nature. We turned our attention to identify the root cause for the formation of bromoclenbuterol in clenbuterol synthetic process. After thorough investigation, it was identified as mono chloro intermediate is the main root cause for the bromoclenbuterol formation.

In the synthesis of clenbuterol hydrochloride, first step was a double chlorination of 4-aminoacetophenone (1) through an electrophillic aromatic substitution reaction to yield 4-amino-3,5dichloroacetophenone (2). Due to the ortho/para directing, amino group and the meta directing, electron withdrawing, acetyl group, chlorination of 4 -aminoacetophenone occurs primarily at the 3 and 5 positions over the 2 and 6 positions. Therefore, under chlorination would produce only the mono chlorinated impurity, 4-amino-3chloroacetophenone. Under these conditions, over chlorination does not result in the addition of chlorine to the 2 and 6 positions because the amino and acetyl groups do not direct that addition. Even though chlorides are ortho/para directing and direct to the 2 and 6 position, chlorides are also deactivating. After close observation on this chlorination reaction, it was noted that the formed mono chlorinated impurity (Scheme 2) (4-amino-3-chloro acetophenone) caused the formation of process related impurity (bromoclenbuterol) in clenbuerol synthesis.

As per the above said observations on chlorination reaction, we concluded that the control of monochloro impurity should be itself in the initial stage i.e., chlorination reaction, by using different crystallization techniques. In the same way, the focus turned on the synthesis of bromoclenbuterol from mono chlorinated impurity by using Scheme 3 and this impurity should be necessary in the method development and validation of clenbuterol hydrochloride API molecule. 4-Amino acetophenone (1) was reacted with $\mathrm{N}$-Chlorosuccinimide in $1 \mathrm{~N} \mathrm{HCl}$ to afford 4-amino-3-chloro acetophenone (7), which was<smiles>CC(=O)c1cc(Cl)c(N)c(CC(C)C(C)=O)c1</smiles>

1
2<smiles>Nc1c(Cl)cc(C(=O)CBr)cc1Cl</smiles>

3<smiles>CC1CCCCC1(C)N</smiles><smiles>CC(=O)CNC(C)C</smiles>

4 $\mathrm{NaBH}_{4}$<smiles>CC(C)(C)NCC(O)c1cc(Cl)c(N)c(Cl)c1</smiles>

Scheme 1: Route of synthesis for clenbuterol.<smiles>CC(=O)c1ccc(N)c(Cl)c1</smiles>

Scheme 2: Chlorination of 4-amino acetophenone. 


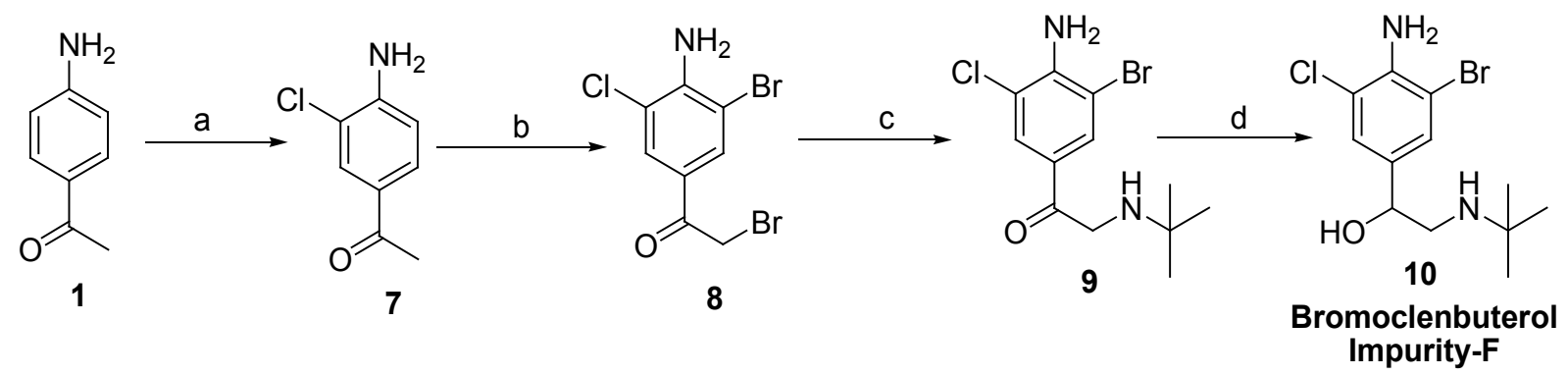

a) $1 \mathrm{~N} \mathrm{HCl}, \mathrm{NCS}$ b) $\mathrm{Br}_{2}, \mathrm{CHCl}_{3}$ c) tert-butylamine, $\mathrm{KI}, \mathrm{CHCl}_{3}$ d) $\mathrm{NaBH}_{4}$, Methanol

Scheme 3: Bromoclenbuterol route of synthesis.

reacted with bromine to give 1-(4-amino-3-bromo-5-chlorophenyl)2-bromoethanone (8). The obtained bromo compound was reacted with tertiay -butyl amine to afford 2-(tert-butylamino)-1-(4-amino-3bromo-5-chlorophenyl)ethanone (9), which was reduced with sodium borohydride in methanol to give bromoclenbuterol compound (10). The synthesized bromoclenbuterol structure was confirmed by ${ }^{1} \mathrm{H}$ NMR, ${ }^{13} \mathrm{C}$ NMR, IR and mass spectra.

\section{Conclusion}

The known impurity, bromoclenbuterol (Impurity-F in clenbuterol as per $\mathrm{BP}$ and $\mathrm{EP}$ ) was synthesized and confirmed by the characterization tools such as HPLC, ${ }^{1} \mathrm{H}$ NMR, ${ }^{13} \mathrm{C}$ NMR, IR and MASS.

\section{Acknowledgments}

The authors are thankful to the management of RA Chem Pharma Limited, for the financial support and encouragement.

\section{References}

1. International Conference on Harmonization (ICH) Guidelines (2002) Q3A (R) Impurities in New Drug Substances.

2. $\mathrm{ICH}(2006)$ Impurities in New Drug Products. Q3B (R1).

3. Srinivasulu R, Ravi Kumar K, Nageswararao K (2016) Synthesis of 3,4-dihydropyrimidin-2(1H)-ones using camphor sulfonic acid as a catalyst under solvent-free conditions. Derpharma chemica 8: 375-379.

4. Antuna AZ, Ivan L, Pablo RG, Julio R, Jose, et al. (2011) V. Tetrahedron 67: 5577-5581.

5. Ehrhardt JD (1990) Synthesis of nonadeutero-clenbuterol. Journal of labeled compounds and radiopharmaceuticals 28: 725-729.

6. Drabb TW (1990) Method for the preparation of $4^{\prime}$-(substituted)-amino-2 (substituted) Amino-3',5'-dichloroacetophenone and salts thereof Trenton. NJ Patent: US4906781A.

7. Bentley TJ (1984) Method for the preparation of 1-(4'-amino-3',5'dichlorophenyl)-2-alkyl(or dialkyl)aminoethanols. US4461914 A. 